Rev Cuid. 2020; 11(1): e784 Open Access $\quad$ ARTÍCULO DE INVESTIGACIÓN

\title{
Retenção de conhecimento dos enfermeiros sobre derivação ventricular externa
}

\author{
Retaining knowledge of external ventricular drain by nursing professionals
}

\section{Retención del conocimiento de los enfermeros sobre derivación ventricular externa}

\author{
Regina Claudia Silva Souza ${ }^{1}$, Ellen Maria Pires Siqueira ${ }^{2}$, Luciana Meira ${ }^{3}$, \\ Giane Leandro Araujo ${ }^{4}$, Mariana Davies Ribeiro Bersaneti ${ }^{5}$
}

\section{Histórico}

Recibido:

20 de febrero de 2019

Aceptado:

10 de octubre de 2019

1 Hospital Sírio Libanês - Núcleo de Novos Conhecimentos, São Paulo, SP-Brasil.

E-mail: Autor de Correspondência. regina.souza@hsl.org.br https://orcid.org/0000-0002-5220-916X

2 Hospital Sirio Libanês - Unidade de Terapia Intensiva, São Paulo, SP-Brasil.

E-mail:ellen.pires@hsl.org.br https://orcid.org/0000-0002-1198-2359

3 Hospital Sírio Libanês Núcleo de Desenvolvimento de Enfermagem, São Paulo, SPBrasil.

E-mail:1 luciana.meira@hsl.org.br https://orcid.org/0000-0003-2840-3584

4 Hospital Sirio Libanês - Unidade de Terapia Intensiva, São Paulo, SP-Brasil.

E-mail: gianeleandro@uol.com.br https://orcid.org/0000-0001-8826-8949

$5 \frac{\text { Hospital Sírio Libanês - Unidade }}{\text { Hot }}$ de Terapia Intensiva, São Paulo, SP-Brasil.

E-mail: mariana.ribeiro@hsl.org.br https://orcid.org/0000-0003-0426-7493
Introdução: A hidrocefalia é uma complicação frequente entre os pacientes neurológicos e seu tratamento em casos agudos é por meio de um cateter de derivação ventricular externa que possibilita a monitorização e o controle da hipertensão intracraniana. O objetivo desse estudo foi descrever a retenção do conhecimento dos enfermeiros após intervenção educativa sobre cuidados com derivação ventricular externa. Materiais e Métodos: Estudo quase experimental com enfermeiros de uma unidade de terapia intensiva adulto, em que foi avaliado a retenção de conhecimento sobre o tema em três momentos: antes, uma semana e três meses após o treinamento. Resultados: Houve retenção significativa após uma semana, mas não aos três meses, sendo que aos três meses o índice de acertos nas questões foi expressivamente baixo em relação às fases anteriores. $\mathrm{O}$ desempenho dos participantes foi melhor nas questões relacionadas ao manuseio do sistema. Discussão: O tempo é um fator que interfere no aprendizado e os estudos mostram que o conhecimento aos 3 meses diminui significativamente, sendo que aos 6 meses e um ano quase que completamente. Condutas práticas baseadas em diretrizes de assistência tem melhor retenção entre os profissionais, ressaltando que há mais facilidade no aprendizado de habilidades técnicas que estão associadas a vivência profissional. Conclusões: Houve retenção de conhecimento significativa entre os profissionais na primeira semana após o treinamento, mas não aos três meses após o treinamento.

Palavras chave: Conhecimento; Capacitação em Serviço; Líquido Cefalorraquidiano, Cuidados de Enfermagem.

Abstract

Introduction: Hydrocephalus is a common complication among neurological patients whose treatment in acute cases is done through an external ventricular drain catheter which allows monitoring and controlling intracranial hypertension This study aimed at describing the knowledge retention by nurses after an educational intervention on nursing care for an external ventricular drain. Materials and Methods: A quasi-experimental study with nurses at an adult intensive care unit was conducted in which knowledge retention on the topic was evaluated at different three times: before, one week and three months after training. Results: After one week, significant retention was observed but not after three months, given that after this period the question success rate was significantly low with respect to the previous phases. Participants' performance was better in those questions related to system management. Discussion: Time is a factor that interferes with learning since studies show that knowledge at three months significantly decreases and is almost completely gone after six months and a year. Practical behaviors based on assistance guidelines have better retention among professionals, underlining that it is easier to learn technical skills that are associated with professional experience. Conclusions: A significant knowledge retention was observed among professionals in the first week after training but not after three months.

Key words: Knowledge; Inservice Training; Cerebrospinal Fluid; Nursing Care.

Resumen

Introducción: La hidrocefalia es una complicación frecuente entre los pacientes neurológicos y su tratamiento en casos agudos se hace a través de un catéter de derivación ventricular externa que permite monitorear y controlar la hipertensión intracraneal. El objetivo de este estudio fue describir la retención del conocimiento de los enfermeros después de la intervención educativa sobre cuidados relativos a la derivación ventricular externa. Materiales y Métodos: Estudio cuasi experimental con enfermeros de una unidad de terapia intensiva de adultos, en el que se evaluó la retención del conocimiento sobre el tema en tres momentos: antes, una semana y tres meses después del entrenamiento. Resultados: Después de una semana se observó una retención significativa, sin embargo no a los tres meses, dado que después de tres meses el índice de aciertos en las preguntas fue significativamente bajo con respecto a las fases anteriores. El desempeño de los participantes fue mejor en las preguntas relacionadas con el manejo del sistema. Discusión: El tiempo es un factor que interfiere en el aprendizaje y los estudios muestran que el conocimiento a los tres meses disminuye significativamente, y después de seis meses y un año casi por completo. Las conductas prácticas basadas en directrices de asistencia tienen mejor retención entre los profesionales, resaltando que resulta más fácil aprender habilidades técnicas que están asociadas con la vivencia profesional. Conclusiones: Se observó una retención de conocimiento significativa entre los profesionales en la primera semana posterior al entrenamiento, pero no a los tres meses después del entrenamiento.

Palabras clave: Conocimiento; Capacitación en Servicio; Líquido Cefalorraquídeo; Atención de Enfermería.

Como citar este artigo: Souza RCS, Siqueira EMP, Meira L, Araujo GL, Bersaneti MDR. Retenção de conhecimento dos enfermeiros sobre derivação ventricular externa. Rev Cuid. 2020; 11(1): e748. http://dx.doi.org/10.15649/cuidarte.784

(c) (1) $(92020$ Universidad de Santander. Este es un artículo de acceso abierto, distribuido bajo los términos de la licencia Creative Commons (c) 1 Attribution (CC BY-NC 4.0), que permite el uso ilimitado, distribución y reproducción en cualquier medio, siempre que el autor original y la fuente sean debidamente citados. 


\section{INTRODUÇÃO}

A hidrocefalia é umas das mais frequentes complicações encontradas nos pacientes nas unidades de neurocirurgia, podendo ocorrer em diferentes situações clinicas $^{1}$. Seu tratamento em casos agudos é por meio da colocação de um cateter de derivação ventricular externa (DVE) que também possibilita a monitorização e o controle da hipertensão intracraniana quando necessário ${ }^{1}$.

Esse tratamento pode trazer aos pacientes o risco de desenvolver infecções como meningites e ventriculites, o que resulta em aumento da morbidade e mortalidade. A incidência dessas infecções é de cerca de $2 \%$ a $27 \%$ e está relacionada com o tempo de permanência do cateter, a doença de base, a técnica de inserção do cateter, o tipo de dispositivo usado para drenagem do líquor e a manipulação do sistema ${ }^{1,2}$.

Diminuir o risco de infecção para esses pacientes impacta em redução de custos pelo menor tempo de hospitalização e uso de recursos; além de contribuir para melhores resultados da assistência. Outro aspecto relevante é a manipulação do sistema e sua associação com eventos adversos geralmente causados por lacunas no treinamento das equipes ${ }^{2}$.

Estudos mostraram que as intervenções educativas com as equipes de saúde refletiram em uma redução de $40 \%$ a $50 \%$ das infecções relacionadas a dispositivos invasivos,$\frac{3,4}{}$. Portanto, implementar ações dessa natureza e promover uma cultura de prevenção de danos é fundamental no cuidado em saúde.

A implementação de cuidados padronizados com DVE segundo guidelines e protocolos e a instrumentalização dos profissionais em relação a essas questões são estratégias reconhecidas na prevenção das infecções referentes a esse dispositivo e também no desenvolvimento de uma cultura de segurança $a^{2,4,5}$.

Os profissionais de saúde estão expostos a uma demanda importante de informações e recursos tecnológicos, o que exige uma qualificação para garantir a segurança do paciente e decisões mais assertivas. Assim, é essencial que esses profissionais possuam conhecimento e habilidades para a realização da assistência. Os treinamentos são cada vez mais usados para ensinar e praticar novas habilidades $\underline{6}$ e na busca contínua por conhecimento, atualização profissional e capacitação.

Há na literatura, uma ampla variedade de teorias de aprendizagem e modelos educacionais para avaliar ensino e aprendizagem, com uma extensa diversidade de estratégias de ensino que podem ser utilizadas para desenvolver as competências cognitivas e as habilidades clínicas necessárias para as práticas de saúde $^{7}$. Essas estratégias são definidas de acordo com os objetivos de aprendizagem, recursos disponíveis e experiência dos educadores. A medida que o conhecimento é aprimorado e a adesão as boas práticas é 
enfatizada, os desfechos em saúde podem ser mais positivos, porém é fundamental entender as necessidades e os resultados de aprendizagem para que possamos implementar estratégias de educação mais eficientes e efetivas.

Em nosso contexto de trabalho, observamos uma necessidade de instrumentalização dos enfermeiros referente aos cuidados com DVE para reforçar aspectos de segurança, qualidade e educação de pacientes e familiares, como também a necessidade de compreender quais fatores favorecem a retenção de conhecimento dos enfermeiros sobre o tema.

Nosso objetivo foi descrever a retenção de conhecimentos dos enfermeiros de uma Unidade de Terapia Intensiva (UTI), após uma intervenção educativa sobre cuidados com Derivação Ventricular Externa e analisar se as variáveis sociodemográficas tem impacto na retenção de conhecimento dos enfermeiros.

\section{MATERIAIS E MÉTODOS}

\section{Delineamento do estudo}

Estudo quase experimental baseado em uma intervenção educativa com duração de 01 mês, com enfermeiros sobre cuidados com DVE, realizado entre os meses de novembro de 2015 a março de 2016. Os enfermeiros foram avaliados antes, uma semana e três meses após o treinamento por meio de um questionário aplicado presencialmente pelos pesquisadores, que continha 10 questões sobre o conteúdo abordado no treinamento.

\section{População e amostra}

Os 38 enfermeiros incluídos no estudo trabalhavam em uma UTI geral adulto de um hospital privado do município de São Paulo, Brasil e participaram do treinamento sobre os cuidados com DVE. Foram excluídos os profissionais que se encontravam no momento do estudo em férias ou licença médica e que não participaram do treinamento.

\section{Procedimento de coleta de dados}

O local do estudo é uma UTI geral com 29 leitos distribuídos em três áreas que se destinam a cuidados a pacientes oncológicos, pôs operatório de cirurgias de grande porte e neurológicos, sendo que o número total de enfermeiros assistenciais da unidade são 50 profissionais.

A intervenção educativa consistiu de uma aula expositiva presencial associada a um vídeo sobre a técnica de mensuração do líquor, curativo e nivelamento do sistema de drenagem de DVE. O conteúdo abordado na estratégia educacional envolveu a fisiopatologia da hidrocefalia e hipertensão intracraniana, complicações e cuidados relativos ao tratamento como manuseio do sistema. A estratégia foi planejada e elaborada pelos pesquisadores de acordo com o Guideline da American Association of Neuroscience Nurses $^{5}$, que estabelece os mais altos padrões 
de atendimento para pacientes com doenças questões se referiam ao conteúdo abordado neurológicas e foi validada por enfermeiros e na intervenção educativa. Assim como a médicos com experiência em neurointensivismo intervenção, o questionário de avaliação e terapia intensiva. A intervenção teve a duração também foi construído e validado pelos mesmos de 60 minutos e foi realizada durante o turno profissionais e seu preenchimento teve a duração de trabalho dos profissionais durante um mês de aproximadamente 30 minutos. Sua aplicação pelos pesquisadores, que são enfermeiros com foi durante o turno de trabalho dos participantes experiência em educação e terapia intensiva. A escolha por essa estratégia foi pela experiência da equipe envolvida no treinamento nessa metodologia de educação e pela otimização do tempo e recursos ${ }^{8,9}$ e em razão de que não há evidência de alta qualidade sobre o tipo de estratégia educacional mais eficaz para a transferência de conhecimento ${ }^{10}$.

Os pesquisadores aplicaram um questionário em três momentos: fase I, antes da realização do treinamento, fase II que aconteceu uma semana após o treinamento e fase III que correspondeu ao período de três meses após o treinamento.

O conhecimento dos enfermeiros quanto ao tema proposto foi mensurado pelo número de questões assinaladas corretamente, lembrando que neste caso, o número de acertos poderia variar de 0 a que continha perguntas sobre características sociodemograficas e dez questões com cinco possíveis respostas para cada, dentre as quais, apenas uma alternativa era a correta. Essas 10 , ou seja, de $0 \%$ a $100 \%$ de acertos.

O estudo foi aprovado pelo Comitê de Ética e Pesquisa (CEP) da instituição sob o número HSL 2015-81. 


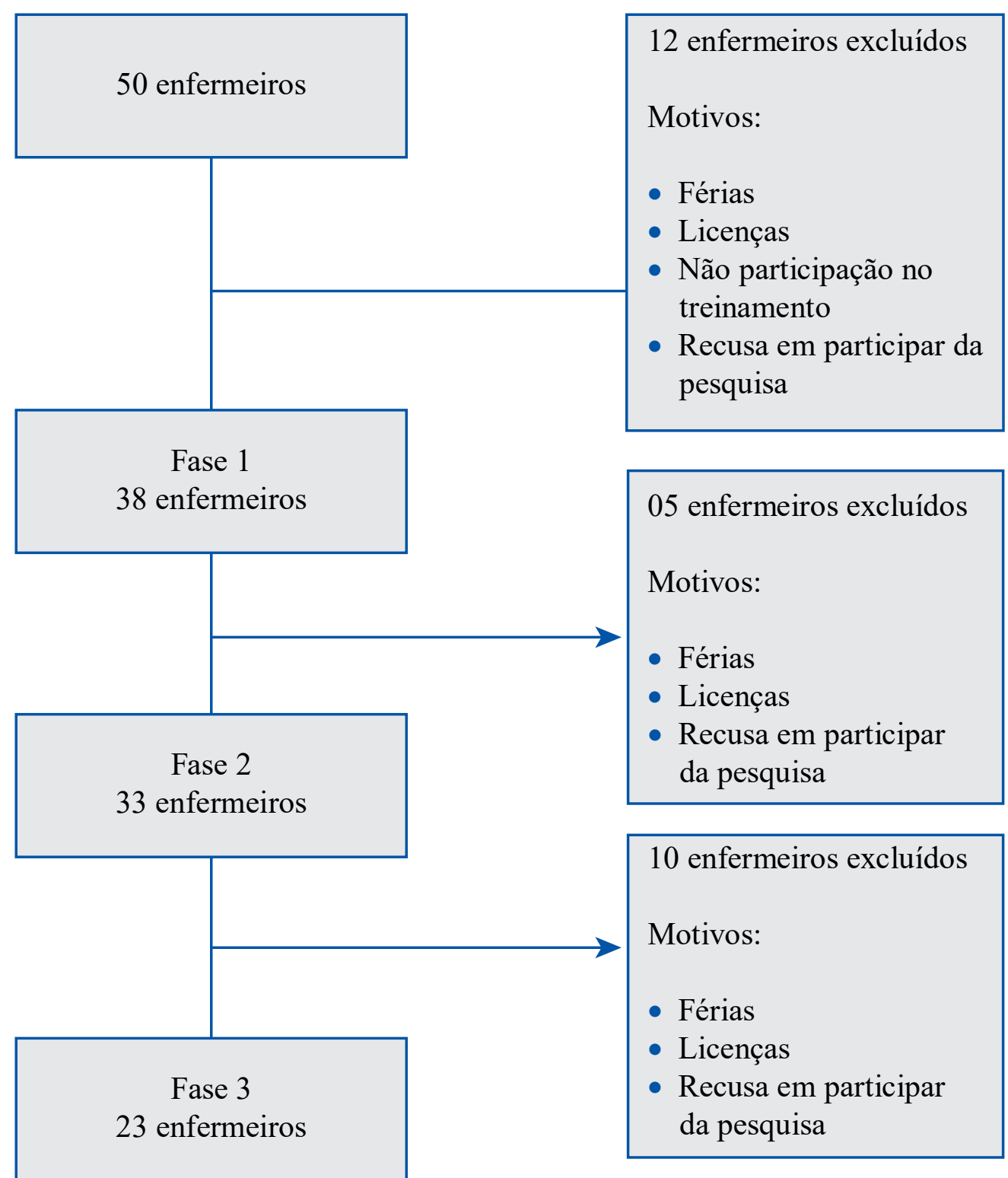

Figura 1- Fluxograma do recrutamento de participantes do estudo. São Paulo, 2018.

\section{Análise de dados}

As análises estatísticas foram realizadas com o programa estatístico SPSS Statistics for Windows, versão 23.0 e a distribuição da amostra foi calculada usando o teste Kolmogorov Smirnov. Para variáveis categóricas, os dados foram expressos em frequência absoluta e relativa e as variáveis quantitativas foram expressas em medidas de tendência central, dispersão e interquartis $(25 \%$ e $75 \%)$. Para comparar os acertos dos sujeitos ao longo das três fases utilizou o teste t de Student pareado e o teste de Friedman para analisar as variáveis relacionadas ao conteúdo do questionário. Para a comparação entre os grupos da retenção do conhecimento com 
as variáveis sociodemográficas não paramétricas foi usado o teste de Kruskal-Wallis com pós teste de Bonferroni e para as categóricas o teste Mann Whitney. Na comparação da porcentagem de acertos ao longo das três fases, utilizou- se o teste Q Cochran. O nível de significância determinado foi $\mathrm{p}<0,05$.

\section{RESULTADOS}

A amostra foi composta por 38 enfermeiros com idade média 33,7 $( \pm 4,8)$ anos. Os enfermeiros com idade entre 20 a 29 anos representaram $21,1 \%$ da amostra e os com idade entre 30 a 39 anos $65,8 \%$ e o gênero feminino foi o que prevaleceu com 33 participantes $(86,8 \%)$. Houve uma perda ao longo do estudo, de 15 participantes devido a rotatividade de funcionários, licenças médicas e baixa adesão na participação no estudo.

Em média, os enfermeiros possuíam 9,5 $( \pm 5)$ anos de formados, com tempo de formação em $50 \%$ da amostra, superior a 10 anos e com duas especializações em $29,7 \%$.

As variáveis sociodemográficas entre os grupos não mostraram diferença significativa no conhecimento geral sobre o tema (Tabela 1).

\section{Tabela 1. Variáveis sociodemográficas em relação ao conhecimento entre os grupos.}

São Paulo, 2018

\section{Variáveis}

Idade

20 a 29 vs 30 a 39

$0,212 *$

20 a 29 vs 40 a 49

$0,448 *$

30 a 39 vs 40 a 49

$0,818^{*}$

Sexo

Feminino vs masculino

$0,756 * *$

Experiência em UTI neurológica

Sim vs não

$0,177 * *$

Tempo de Formação

$<10$ anos vs $>=10$ anos

$0,697 * *$

Número de especializações

Uma vs Duas ou mais

$0,117 * *$

*Teste de Kruskal-Wallis com pós teste de Bonferroni, **Mann-Whitney.

A retenção de conhecimento mostrou-se primeira e terceiras fases (p-0,20), sendo que a significativa entre a primeira e segunda fases (p- pontuação dos acertos obteve média de $4( \pm 1,3)$ e $0,004)$ e não mostrou diferença relevante entre a $5( \pm 1,4)$ (Tabela 2$)$. 
Tabela 2. Número de acertos do questionário pelos enfermeiros de acordo com a fase do estudo. São Paulo, 2018

\begin{tabular}{cccc} 
& Fase $\mathbf{1}$ & Fase $\mathbf{2}$ & Fase 3 \\
Media & 4 & 5 & 5 \\
Desvio padrão & 1,3 & 1,3 & 1,4 \\
Mínimo - máximo & $2-6$ & $2-8$ & $1-7$ \\
$\mathrm{p}$ & & 0,004 & 0,20 \\
\hline
\end{tabular}

As questões relacionadas a fisiopatologia (Tabela 3). obtiveram mediana menor em relação aos outros conteúdos em todas as fases, com p-0,059 entre a fase 1 e 2 . O conteúdo sobre o manuseio do sistema, apresentou a melhor performance entre os participantes, especialmente entre a fase 1 e 2 com p-0,001, sendo que entre essas duas fases, o

Os enfermeiros com idade entre 20 e 29 anos demonstraram melhor desempenho p-0,040 com mediana de acertos de 20 (0-40) nas questões relacionadas ao manuseio do sistema quando comparado ao grupo entre 30 e 39 anos. desempenho geral foi significativo com p- 0,014

Tabela 3. Retenção de conhecimento dos enfermeiros de acordo com o conteúdo e a fase do estudo. São Paulo, 2018

$\begin{array}{cccc}\text { Tempo Fase } 1(n=38) & \text { Fase } 2(n=33) & \text { Fase } 3(n=23) & \begin{array}{c}\text { Teste de Friedman } \\ \text { com pós teste de } \\ \text { Muller }- \text { Dunn }\end{array}\end{array}$

Conteúdo n Mediana 25\% 75\% n Mediana 25\% $75 \%$ n Mediana 25\% $75 \% 1$ vs 21 vs 32 vs 3 das questões

\begin{tabular}{|c|c|c|c|c|c|c|c|c|c|c|}
\hline $\begin{array}{c}\text { Especificidades } \\
(\%)\end{array}$ & 38 & 50,0 & 50,0 & 100,033 & 100,0 & 50,0 & 100,023 & 100,0 & 50,0 & $\begin{array}{llll}100,0 & 0,532 & 0,527 & 0,480\end{array}$ \\
\hline $\begin{array}{c}\text { Fisiopatologia } \\
(\%)\end{array}$ & 38 & 33,3 & 33,3 & $41,7 \quad 33$ & 33,3 & 33,3 & $66,7 \quad 23$ & 33,3 & 33,3 & $\begin{array}{llll}33,3 & 1,000 & 0,059 & 0,096\end{array}$ \\
\hline $\begin{array}{c}\text { Manuseio } \\
(\%)\end{array}$ & 38 & 40,0 & 20,0 & $40,0 \quad 33$ & 40,0 & 40,0 & 60,023 & 40,0 & 40,0 & $\begin{array}{llll}60,0 & 0,001 & 0,196 & 0,723\end{array}$ \\
\hline Total $(\%)$ & 38 & 40,0 & 30,0 & $50,0 \quad 33$ & 50,0 & 50,0 & 60,023 & 50,0 & 40,0 & $\begin{array}{llll}50,0 & 0,014 & 0,388 & 0,337\end{array}$ \\
\hline
\end{tabular}

As questões relacionadas ao manuseio e menor na fase I quando comparada as demais manutenção do sistema obtiveram melhor fases $(p=0,066)$. Para as demais questões não foi performance dos participantes quando possível evidenciar mudança estatisticamente comparadas as demais, mas somente, a questão 10 significante ao longo do tempo (Tabela 4). obteve uma tendência da porcentagem de acertos 
Tabela 4. Distribuição do desempenho dos enfermeiros, segundo questão e fase de avaliação. São Paulo, 2018

Domínio

Fisiopatologia

Manuseio

Especificidades

Questão 1- Tratamento inicial da hipertensão intracraniana Questão

Questão 2- Indicações da Derivação Ventricular Externa (DVE)

Questão 3- Fatores de risco para infecções nos pacientes que usam DVE

Questão 6- Complicações da monitorização da PIC

Questão 4- Boas práticas de monitorização da Pressão Intracraniana (PIC) e drenagem do liquor

Questão 7- Boas práticas de curativo do cateter de DVE

Questão 9- Boas práticas na inserção do cateter de DVE

Questão 10- Boas práticas na remoção do cateter de DVE

Questão 5- Localização anatômica cateteres de monitorização de pressão intracraniana

Questão 8- Competências dos profissionais relativas ao manejo do sistema de DVE

\section{Fase 1 Fase 2 Fase 3 pa}

$63,2 \% \quad 84,8 \% \quad 87,0 \% \quad 0,156$

$23,7 \% \quad 36,4 \% \quad 34,8 \% \quad 0,641$

$68,4 \% \quad 75,8 \% \quad 78,3 \% \quad 0,882$

$5,3 \% \quad 36,4 \% \quad 13,0 \% \quad 0,066$

$76,3 \% \quad 84,8 \% \quad 87,0 \% \quad 0,368$

$2,6 \% \quad 12,1 \% \quad 4,3 \% \quad>0,999$

${ }^{\mathrm{a}} \mathrm{Q}$ de Cochran

\section{DISCUSSÃO}

A eficácia das estratégias de educação tem sido objeto de vários estudos pela sua importância e impacto na adesão dos profissionais de saúde aos guidelines e consequentemente em melhor desempenho dos resultados dos cuidados em saúde ${ }^{11}$. Identificar a melhor maneira de alcançar esses propósitos é essencial na qualidade da assistência à saúde e também para a educação médica ${ }^{12}$.

Nosso estudo avaliou a retenção de conhecimento de enfermeiros de uma UTI sobre derivação 
ventricular externa após a realização de um treinamento que se baseou em uma intervenção educativa. Os resultados mostraram que houve retenção de conhecimento dos profissionais significativa após uma semana, mas não após três meses. Podemos considerar que o tempo, é um fator que interfere no aprendizado; o que vem sendo descrito nos estudos; principalmente aos três meses após os treinamentos e intervenções de educação, sendo que aos 6 meses e um ano, a retenção de conhecimento é quase nula ${ }^{7}$.

Conhecimento e habilidades quando não utilizadas, se tornam obsoletas, reforçando que o conhecimento associado a prática é mais significativo, fato observado em nossos resultados com o conteúdo relacionado as boas práticas de manuseio do sistema. Estudantes de enfermagem antes e após a vivência de aprendizagem por três estratégias diferentes de ensino, obtiveram redução significativa da retenção do conhecimento $\frac{13}{}$ após um mês da intervenção. Esse aspecto fortalece a necessidade das atualizações e capacitações frequentes para os profissionais ${ }^{14}$, particularmente em conteúdos mais complexos, como os relacionados a neurointensivismo. Em nosso estudo, a retenção de conhecimento na terceira fase foi muito baixa, mas a adesão foi comprometida pela perda de 15 participantes, o que pode ter influenciado os resultados.

Mesmo, com resultados nem sempre satisfatórios; os estudos com intervenção educativa entre os enfermeiros apresentam bons resultados em relação a melhora do desempenho desses profissionais no que diz respeito ao conhecimento, destacando que essas estratégias são essenciais para um bom desempenho profissional e resultados positivos de assistência ${ }^{15,16,17}$. No que se refere ao tema de nosso estudo, esse pressuposto também foi confirmado por diminuição das taxas de infecção em outras pesquisas $\stackrel{2,4}{ }$.

As variáveis sociodemográficas não influenciaram a retenção de conhecimento, com exceção da faixa etária de 20 a 29 anos, no conteúdo relacionado as boas práticas no manuseio do sistema. Os estudos mostram que não há correlação entre as características individuais e o conhecimento, mas há associação entre essas variáveis e as atitudes relacionadas aos cuidados em saúde ${ }^{17}$. Kassa et al, em sua pesquisa, sobre avaliação do conhecimento, atitudes e práticas em cuidados paliativos identificou que a idade, o gênero, o nível de educação e a experiência profissional se associaram somente as atitudes sobre o tema ${ }^{17}$. Esse resultado, contrasta com nosso estudo, em relação ao conhecimento, em que os profissionais mais jovens apresentaram melhor desempenho que os demais; mas que é validado por uma nova perspectiva, onde os enfermeiros novatos desenvolvem habilidades no pensamento crítico e alcançam as competências desejadas mais rápido por meio de treinamentos e preceptoria no contexto da pratica, facilitando o seu progresso profissional $\frac{18}{}$.

Um dado relevante é que as questões sobre fisiopatologia, não obtiveram bons resultados nas fases do estudo, o que chama a atenção, porque o conhecimento sobre fisiopatologia é fundamental na tomada de decisão clínica, uma vez que os enfermeiros precisam saber integrar a evidência 
cientifica com as necessidades dos pacientes, o contexto clinico e os recursos disponíveis $\frac{19}{}$. Uma lacuna em conhecimento dessa natureza, impacta diretamente nos resultados do cuidado, sendo essencial uma melhor performance dos enfermeiros nesse sentido para promover as mudanças no sistema de saúde $\frac{19}{}$.

É reconhecido que o trabalho dos enfermeiros em contextos diversificados e em um momento atual de transição global da saúde, com avanços científicos e tecnológicos; precisa estar associado a um compromisso desse profissional com aprendizagem nas ciências biológicas ao longo da vida, porque essas são relevantes para os cuidados de saúde e imperativas para cuidados de enfermagem eficazes ${ }^{20}$. Corroborando esse aspecto, a enfermagem tem um papel importante a desempenhar na concepção e prestação de serviços de saúde eficazes e eficientes para as populações do mundo ${ }^{21}$.

O conteúdo sobre o manuseio do sistema foi o que apresentou melhores resultados quanto a retenção do conhecimento. São condutas práticas, baseadas em diretrizes de assistência; ressaltando que os profissionais possuem mais facilidade com habilidades técnicas e que estão associadas com a experiência profissional. Em estudo realizado com enfermeiros portugueses, foi evidenciado que os enfermeiros mais novos, com 0 a 5 anos de experiência profissional apresentaram escores médios mais baixos de competência profissional, enquanto os outros grupos etários apresentam todos valores mais elevados 22 .
O estudo traz contribuições para a prática, ao observarmos que conteúdos relacionados a educação em saúde precisam ser abordados de forma contínua e sistematizada com o uso de estratégias que permitam a aplicação do conhecimento juntamente com as habilidades técnicas, desenvolvendo o raciocínio clínico e a competência das pessoas.

\section{Limitações do estudo}

Nosso estudo encontrou algumas limitações como uma perda expressiva de participantes ao longo das fases e o treinamento durante o turno de trabalho que podem ter influenciado os resultados.

\section{CONCLUSÕES}

O estudo mostrou que a intervenção educativa proposta e baseada em uma estratégia de aula expositiva e vídeo foi efetiva na retenção de conhecimento entre os enfermeiros, mas o tempo pode ter sido um fator que contribuiu para diminuição de retenção na terceira fase. Esse resultado implica em implementar e avaliar estratégias inovadoras de treinamento, ou mesmo associá-las para alcançar melhores resultados ao longo do tempo. É fundamental ampliar as pesquisas referentes a essa temática com outras populações.

Conflito de interesses: Os autores declaram que não houve conflitos de interesse. 


\section{REFERENCIAS}

1. Lwin S, low SW, Choy DKS, Yeo TT, Chou N. External ventricular drain infections: successful implementation of strategies to reduce infection rate. Singapore Med J. 2012; 53(4): 255-9.

2. Camacho EF, Boszczowski I, Freire MP, Pinto FC, Guimaraes T, Teixeira MJ et al. Inpact of an educational implanted in a neurological intensive care unit on rates of infection related to external ventricular drains. PLOS ONE. 2013; 8(2): e50708.

https://doi.org/10.1371/journal.pone.0050708

3. Lobo RD, Levin AS, Gomes LM, Cursino R, Park M, Figueiredo VB et al. Impact of an educational program and policy changes on decreasing catheter-associated bloodstream infections in a medical intensive care unit in Brazil. Am J Infect Control. 2005; 33(2): 83-7.

https://doi.org/10.1016/j.ajic.2004.05.003

4. Dasic D, Hanna Sj, Bojanic S, Kerr RS. External ventricular drain infection: the effect of a strict protocol on infection rates and a review of the literature. Br J Neurosurg. 2006; 20(5): 296-300.

https://doi.org/10.1080/02688690600999901

5. American Association of Neuroscience Nurses. Care of the Patient Undergoing Intracranial Pressure Monitoring/ External Ventricular Drainage or Lumbar Drainage. Glenview: AANN Clinical Practice Guideline; 2011. 38p. [cited 2018 fev 15]. Available from:

http://www.aann.org/uploads/AANN11_ICPEVDnew.pdf

6. Kamal AH, Quinn D, Gilligan TD, Davis BC, Dalby CK, Bretsch J et al. ReCAP: Feasibility and Effectiveness of a Pilot Program to Facilitate Quality Improvement Learning in Oncology: Experience of the American Society of Clinical Oncology Quality Training Program. $J$ Oncol Pract. 2016; 12(2): e215-23. https://doi.org/10.1200/JOP.2015.004762

7. Hulse AL. Designing and evaluating vascular access training using educational theory. Br J Nurs. 2018; 25; 27(2): S27-S33. https://doi.org/10.12968/bjon.2018.27.2.S27

8. Lobos BM, Vergara NF. Efecto de un programa de intervención educativa sobre el conocimiento de seguridad de pacientes em estudiantes de pregrado de enfermería. Cienc. enferm. 2017; 23(1): 97-108. https://doi.org/10.4067/S0717-95532017000100097

9. Carvalho LR, Domingues NA, Zem-Mascarenhas SH. Desenvolvimento de tecnologia digital educacional sobre monitoração da pressão intracraniana minimamente invasiva. Texto Contexto Enferm. 2017; 26(4): e0830017. https://doi.org/10.1590/0104-07072017000830017

10. Gordon M, Findley R. Educational interventions to improve handover inhealth care: a systematic review. Medical Education. 2011:45: 1081-9.

https://doi.org/10.1111/j.1365-2923.2011.04049.x

11. Del Cura-González, López-Rodríguez JA, Sanz-Cuesta T, Rodríguez-Barrientos R, Martín-Fernández J, Ariza-Cardiel G, et al. Effectiveness of a strategy that uses educational games to implement clinical practice guidelines among Spanish residents of family and com- munity medicine (e-EDUCAGUIA project): a clinical trial by clusters. Implement Sci. 2016; 17;11: 71.

https://doi.org/10.1186/s13012-016-0425-3

12. Liabsuetrakul T, Sirirak T, Boonyapipat S, Pornsawat P. Effect of continuous education for evidence-based medicine practice on knowledge, attitudes and skills of medical students. J Eval Clin Pract. 2013; 19 (4): 607-11. https://doi.org/10.1111/j.1365-2753.2012.01828.x

13. PourghazneinT, SabeghiH, ShariatinejadK. Effects of e-learning, lectures, and role playing on nursingstudents' knowledge acquisition, retention and satisfaction. Med $J$ Islam Repub Iran. 2015; 25;29: 162. eCollection 2015.

14. Sankar J, Vijayakanthi N, Sankar MJ, Dubey N. Knowledge and Skill Retention of In-Service versus Preservice Nursing Professionals following an Informal Training Program in Pediatric Cardiopulmonary Resuscitation: A Repeated-Measures Quasiexperimental Study. Biomed Res Int. 2013; 2013: 403415. https://doi.org/10.1155/2013/403415

15. Baron MV, Reuter CP, Burgos MS, Cavalli V, Brandenburg C, Krug SBF. Experimental study with nursing staff related to the knowledge about pressure ulcers. Rev Lat Am Enfermagem. 2016; 24: e2831. https://doi.org/10.1590/1518-8345.1134.2831

16. Lazarini FM, Barbosa DA. Educational intervention in Primary Care for the prevention of congenital syphilis. Rev Lat Am Enfermagem. 2017; 30; 25: e2845. https://doi.org/10.1590/1518-8345.1612.2845

17. Kassa H, Murugan R, Zewdu F, Hailu M, Woldeyohannes D. Assessment of Knowledge, attitude and practice and associated factors towards palliative care among nurses working in selected hospitals, Addis Ababa, Ethiopia. BMC Palliat Care. 2014; 13(1):6. https://doi.org/10.1186/1472-684X-13-6

18. Forneris S, Peden-McAlpine C. Contextual Learning: A reflective Learning Intervention for Nursing Education. Int J Nurs Educ Scholarsh. 2006; 3. https://doi.org/10.2202/1548-923X.1254

19. Canadian Nurses Association. Position statement: Evidence-informed decision-making and nursing practice. (2013).

20. Steering Committee of Higher Education Academy Biosciences in Nursing Education (BiNE) Specialist Reference Group. Learning outcomes for biosciences in pre-registration nursing programmes: Quality Assurance Framework for Bioscience Education in Nursing. 2016. [Cited 2018 Apr. 20]. Available in:

https://www.heacademy.ac.uk/.../bioscience-nurse-education-bine

21. Kitson, A. The state of the art and science of evidencebased nursing in UK and Europe. Worldviews Evid Based Nurs. 2004; 1(1): 6-8. https://doi.org/10.1111/j.1741-6787.2004.04010.x

22. Oliveira LMN, Queirós PJP, Castro FV. A competência profissional dos enfermeiros. Um estudo em hospitais portugueses. International Journal of Developmental and Educational Psychology. INFAD Revista de Psicología. 2015; 2(1): 143-58.

https://doi.org/10.17060/ijodaep.2015.n2.v1.331 